PS1 - 189

doi:10.1017/cjn.2016.350

\author{
A Universal Predictive Model for Dose Fall-Off in MLC-Based \\ Stereotactic Brain Radiosurgery

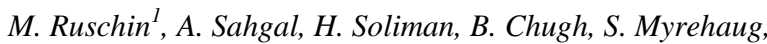 \\ M. Tsao, A. Sarfehnia, C. Yeboah, Y. Lee \\ ${ }^{I}$ University of Toronto, Toronto, ON \\ Mark.Ruschin@sunnybrook.ca
}

Predictive modeling of dose fall-off in radiosurgery could assist in clinical decision-making when prescribing a treatment plan with minimized toxicity risk. The purpose of this study is to develop a predictive dose fall-off model. Materials/Methods: We retrospectively reviewed treatment plans from 257 patients $(365$ lesions) with total doses ranging from 20 to $35 \mathrm{~Gy}$ in 5 fractions. For each plan, we measured both total volume of the external contour (EXT) and BrainMinusPTV (BMP) receiving $\mathrm{P}=20 \%$ to $\mathrm{P}=80 \%$ of the prescription dose. The model has form $\mathrm{y}=\mathrm{Fa}(\mathrm{PTV}) \mathrm{b}+$--delta. $\mathrm{y}=$ volume of EXT or BMP (cc's); $\mathrm{a}$ and $\mathrm{b}$ are curve-fitting coefficients; PTV=total planning target volume (cc's); $F$ is an adjustment factor $(>1)$ to account for number of targets; delta is the $95 \%$ prediction band. F, a, b, and delta were modeled such that dose-fall can be forecast for any PTV and dose level. Results: The model coefficients were as follows: Coefficient EXT BMP a 19927(100 $\times \mathrm{P}) \exp (-2) \quad 17122(100 \times \mathrm{P}) \exp (-2) \quad$ b $0.42(100 \times \mathrm{P}) \exp (0.17) \quad 0.63 \quad \mathrm{~F} \quad-0.0156 \times(100 \times \mathrm{P})+2.5517$ delta $384467 \times(100 \times \mathrm{P}) \exp (-2.3159)$ The table can be used to determine the model for any $\mathrm{P}$ from $20 \%$ to $80 \%$. Example: the EXT receiving $50 \%, \mathrm{P}=0.5, \mathrm{a}=8.0, \mathrm{~b}=0.82, \mathrm{~F}=1.8$, delta $=45$. Thus, EXT$50=8(\mathrm{PTV} 0.82)$ or $1.8 \times 8(\mathrm{PTV} 0.82)$ for $1-3$ or $>3$ targets, respectively, $+/-45 \mathrm{cc}$ 's. The model was verified against published values of dose fall-off from linacs. Conclusion: A predictive dose fall-off model was generated for linac-based radiosurgery. The model can be used for quality assurance or for inter-institutional comparisons. Ongoing work is being conducted to extend the model to a SRS cones system.

\section{QUALITY OF LIFE}

\section{PS1 -125}

doi:10.1017/cjn.2016.351

\section{Assessment of Function and Quality of Life in a Phase II Multi- Institutional Clinical Trial of Fractionated Simultaneous In- Field Boost Radiotherapy for Patients with 1-2 Brain Metastases}

\footnotetext{
G. Bauman I, S. Yartsev ${ }^{2}$, D. Roberge ${ }^{3}$, R. MacRae ${ }^{4}$,W. Roa ${ }^{5}$, V. Panet-Raymond ${ }^{6}$, L. Masucci ${ }^{3}$, B. Yaremko ${ }^{2}$, D. D'Souza ${ }^{2}$, D. Palma ${ }^{2}$, T. Sexton ${ }^{2}$, E. Yu ${ }^{2}$, J.R. Pantarotto ${ }^{4}$, B. Ahmad ${ }^{2}$, B. Fisher ${ }^{2}$, A.R. Dar ${ }^{2}$, C. Lambert ${ }^{3}$, G. Pond ${ }^{7}$, L. Stitt ${ }^{2}$, K.Y. Tay ${ }^{8}$, G. Rodrigues ${ }^{2}$

${ }^{1}$ Division of Radiation Oncology, London Health Sciences Centre, A4-901B, 790 Commissioners Rd. E, London, ON

${ }^{2}$ Division of Radiation Oncology, London Health Sciences Centre, A4-901B, 790 Commissioners Rd. E, London, ON

${ }^{3}$ Department of Radiation Oncology, Centre Hospitalier de l'Université de Montréal, Montreal, QC
}

${ }^{4}$ Division of Radiation Oncology, University of Ottawa, Ottawa, ON

${ }^{5}$ Department of Radiation Oncology, Cross Cancer Institute, Edmonton, $A B$

${ }^{6}$ Department of Radiation Oncology, McGill University Health Centre, Montreal, $Q C$

${ }^{7}$ Department of Oncology, Department of Oncology, McMaster University, Hamilton, ON

${ }^{8}$ Department of Diagnostic Radiology, London Health Sciences

Centre, London, $O N$

glenn.bauman@lhsc.on.ca

We examined functional outcomes and quality of life of whole brain radiotherapy (WBRT) with integrated fractionated stereotactic radiotherapy boost (FSRT) for brain metastases treatment. Methods Eighty seven people with 1-3 brain metastases were enrolled on this Phase II trial of WBRT $(30 \mathrm{~Gy} / 10)+$ simultaneous FSRT, (60Gy/10). Results Mean (Min-Max) baseline KPS, Mini Mental Status Exam (MMSE) and FACT-BR quality of life were 83 (70-100), 28 (21-30) and 143 (98-153). Lower baseline MMSE (but not KPS or FACT-Br) was associated with worse survival after adjusting for age, number of metastases, primary and extra-cranial disease status. Crude rates of deterioration ( $>10$ points decrease from baseline for KPS and FACT-Br, MMSE fall to <27) ranged from $26-38 \%$ for KPS, 32 $59 \%$ for FACT-Br and $0-16 \%$ for MMSE depending on the timepoint assessed with higher rates generally noted at earlier time points $(<6$ months post-treatment). Using a linear mixed models analysis, significant declines from baseline were noted for KPS and FACT-Br (largest effects at 6 weeks to 3 months) with no significant change in MMSE. Conclusions The effects on function and quality of life of this integrated treatment of WBRT + simultaneous FSRT were similar to other published series combining WBRT+SRS.

PS1 - 127

doi:10.1017/cjn.2016.352

A Mixed Method Study of a Peer Support Intervention for Newly Diagnosed Primary Brain Tumour Patients

\section{R. Cashman ${ }^{1}$ \\ ${ }^{1}$ University of British Columbia, Vancouver, BC rcashman@bccancer.bc.ca}

The purpose of this study was to investigate the impact of an intervention designed to support newly diagnosed primary brain tumour patients. The intervention involved a structured, one time meeting between newly diagnosed patients and trained volunteer "veteran" primary brain tumour patients. Methods Two trained volunteers met for a single, face to face meeting on an individual basis with a total of 10 newly diagnosed patients. A combination of questionnaires and interviews were used to investigate the impact of the intervention for the new patients and the volunteers. Results: The intervention appeared to be of substantial value for both groups of participants. Analysis revealed that the newly diagnosed patients experienced a range of benefits, including those related to the themes of: increased hope; valued guidance; hearing what it's really like; overcoming aloneness; and realignment of priorities. Veteran patients experienced a sense accomplishment and decreased anxiety as a result of the intervention. Only minor adverse effects and challenges were reported. Conclusions The findings provide initial evidence that the experimental intervention 
has the potential to be a safe, effective means of promoting psycho-social well-being in newly diagnosed patients and may also have positive effects for veteran patients. Further investigation into the use of one to one, peer support for brain tumour patients is an important research priority.

PS1 - 160

doi:10.1017/cjn.2016.353

\section{A Telephone Based Cognitive Assessment Tool for Brain Metastases Patients}

M.N. Tsao ${ }^{I}$, K. Edelstein, L.J. Bernstein, J. Wong, N. Laperriere, J.R. Perry, A. Sahgal, C. Menard, H. Soliman, E. Chow, T. Barnes, C. Danjoux, B.A. Millar, R. Wong, W. Wells, R.S. McIntyre, W. Mason, C. Chung

${ }^{1}$ University of Toronto, Toronto, ON may.tsao@sunnybrook.ca

Improving neurocognitive outcomes following treatment for brain metastases have become increasingly important. We propose that a brief telephone-based neurocognitive assessment may improve follow-up cognitive assessments in this palliative population. Aim: To prospectively assess the feasibility and reliability of a telephone based brief neurocognitive assessment compared to the same tests delivered face-to-face. Methods: Brain metastases patients to be treated with whole brain radiotherapy (WBRT) were assessed using a brief validated neurocognitive battery at baseline, at 1 month and 3 months following WBRT (in person and over the phone). The primary outcome was feasibility and inter-procedural (in person versus telephone) reliability. The secondary objective was to evaluate the change in neurocognitive function before and after WBRT. Results: Out of 39 patients enrolled, $82 \%$ of patients completed the baseline in-person and telephone neurocognitive assessments. However, at 1 month, only $41 \%$ of enrolled patients completed the in-person and telephone cognitive assessments and at 3 months, only $10 \%$ of patients completed them. Results pertaining to reliability and change in neurocognitive function will be updated. Conclusion: The pre-defined definition of feasibility (at least $80 \%$ completion for face to face and telephone neurocognitive assessments) was met at baseline. However, a large proportion of participants did not complete either telephone or in person neurocognitive follow-up at 1 month and at 3 months postWBRT. Attrition remained a challenge for neurocognitive testing in this population even when a telephone-based brief assessment was used.

PS1 - 163

doi:10.1017/cjn.2016.354

\section{Engaging in Difficult Discussions with Neuro-Oncology Patients: A Case Conference Based Quality Improvement Initiative}

C. Mueller ${ }^{\text {, }}$ D.B. Bilodeau, A.C. Chakraborty, J.E. Ellis, L.G. Gibson, E.I. Isenberg-Grzeda, C.M. Moroney, J.M. Myers, A.S. Scalco, A.S. Speke

${ }^{I}$ Sunnybrook Health Sciences Centre, Toronto, ON christina.mueller@sunnybrook.ca

Due to the non-curative nature of high grade brain tumours "difficult discussions" about goals of care, advance care planning, palliative care, and end of life are inherent to the practice of the neuro-oncology team. Clinician and patient barriers are common and may include anxiety about destroying hope, lack of readiness to discuss end of life topics, difficulty managing emotional responses, and concerns over competency in facilitating difficult discussions. Nonetheless, clinician comfort and skill in facilitating these discussions is critical and can impact patient perception of illness, coping response, and ability to make decisions about care. Methods At our regional cancer centre, a novel quality improvement initiative was designed to bring monthly case presentation of "difficult discussions" into a pre-existing weekly multi-disciplinary case conference (MCC). We will describe the rationale and developmental processes behind this initiative. Roughly 15 neuro-oncology clinicians attended each case conference, with guest attendance from palliative care and psychosocial oncology. Clinician groups represented included physicians $(77 \%)$, nurses $(8 \%)$, nurse practitioners $(10 \%)$, and occupational therapists (5\%). Baseline and monthly surveys were administered to determine clinicians' self- rated practices, skills, and attitudes towards "difficult discussions". Results Early findings indicate that the initiative has been well-received. Physicians indicated highest levels of agreement with the statement "I feel that having difficult discussions is part of my responsibility". Non physician groups indicated the greatest agreement that the intervention is beneficial to their practice. Discussion: Our challenges and successes may help guide others to incorporate a similar initiative at disease-site meetings.

PS1 - 188

doi:10.1017/cjn.2016.355

\section{Rehabilitation Consultation: An Integrated Model for Addressing Rehabilitation Concerns in the Primary Brain Tumor Population}

\author{
I. Lax ${ }^{1}$, M. Daniels, C. Kanter, W. Mason, K. Edelstein \\ ${ }^{I}$ University of Toronto, Toronto, $\mathrm{ON}$ \\ ilyse.lax@uhn.ca
}

Individuals with primary brain tumors experience a range of physical, cognitive and psychosocial sequelae which impact their independence, safety and quality of life. These impairments may be addressed through rehabilitation intervention. Despite acknowledgement that timely rehabilitation services over the course of the disease process is of benefit, few outpatient neurooncology treatment teams include a rehabilitation professional. Purpose: The aims are: (1) to describe a rehabilitation consultation model of care integrated into outpatient neuro-oncology treatment for individuals with primary brain tumors; and (2) to describe the characteristics of individuals referred for rehabilitation services. Methods: This retrospective descriptive study examined data from 200 individuals that received rehabilitation consultation from January 2015 to March 2016 at Princess Margaret Hospital, Pencer Brain Tumor Centre. Information on patient demographics, referral characteristics, and number of patient care visits was collected. Descriptive statistics were calculated. Preliminary Results: Of all patients, $(n=195)$, the most common diagnosis is glioblastoma, $39 \%(\mathrm{n}=76)$, and $50 \%$ are $50-69$ years of age $(\mathrm{M}=55, \mathrm{SD}=15.0)$. The most common reason for initial referral was decline in physical functioning, strength and balance (41\%). In $77 \%$ of cases, patients were seen immediately at the time of referral. In total, 540 consultations were completed (face-to-face $=230$, telephone $=$ 310 ) with 2.78 on average ( $\mathrm{SD}=4.0)$ per patient. Conclusion: Given the range of symptoms that individuals with primary brain 